\title{
氮杂糖修饰的葸醌衍生物的合成及细胞毒性
}

\author{
张平竹魏笑刘欢王齐伟 \\ 马趁 戎瑞雪 李小六* \\ (河北大学化学与环境科学学院 河北省化学生物学重点实验室 保定 071002)
}

\begin{abstract}
摘要 为开发低毒、高效的葱醌类抗肿瘤化合物，设计并利用 $N$-烷氨基氮杂糖与大黄酸的缩合反应，及 $N$-烷氨基氮杂 糖与 1-氨基-4-溴葱酮-2-磺酸的微波促进 $\mathrm{Cu}^{0}$ 催化乌尔曼偶联(Ullmann coupling)反应，合成系列新型氮杂糖修饰的葱醌 衍生物. 对所合成的化合物进行了初步体外肿瘤细胞(Hela、A549、MCF-7 和 MGC-803)的细胞毒性测试.

关键词 蒽醌; 大黄酸; 氮杂糖; 乌尔曼偶联; 细胞毒性
\end{abstract}

\section{Synthesis and Cytotoxicity of Azasugar Modified Anthraquinone Derivatives}

\author{
Zhang, Pingzhu Wei, Xiao Liu, Huan Wang, Qiwei \\ Ma, Chen Rong, Ruixue Li, Xiaoliu* \\ (Key Laboratory of Chemical Biology of Hebei Province, School of Chemistry and Environmental Science, \\ Hebei University, Baoding 071002)
}

\begin{abstract}
In order to develop the anti-tumor agents with high efficiency and low toxicity, a series of novel azasugar modified anthraquinone derivatives have been designed and synthesized by the ammonolysis of $N$-alkylamino azasugar with rhine and microwave assisted Ullmann coupling reaction of $N$-alkylamino azasugar with 1-amino-4-bromoanthraquinone-2-sulfonic acid. Their cytotoxic activities against Hela, A549, MCF-7 and MGC-803 cells were preliminarily evaluated.

Keywords anthraquinone; rhein; azasugar; Ullmann coupling; cytotoxicity
\end{abstract}

葸醌类抗肿瘤药物, 如阿霉素、表阿霉素、米托葱 醌(图 1)等因其抗肿瘤谱广, 广泛用于现代临床肿瘤治 疗上, 为目前临床最重要、应用最广的抗肿瘤药物类型 之一. 但其毒副作用, 例如骨髓造血功能毒性、心脏毒 性等，在一定程度上限制了其进一步的使用 ${ }^{[1,2]}$, 因而开 发葱醌类高效低毒抗肿瘤药物, 仍为抗肿瘤药物开发的 重要任务之一.

大黄酸(Rhein, 4,5-二羟基葱醌-2-甲酸)(图 1)是一种 从传统草药大黄根茎中提取的主要生物活性物质之 - ${ }^{[3]}$, 研究表明其对多种肿瘤细胞, 如人乳腺癌、结肠 癌、肺癌、胶质瘤等癌细胞增殖具有抑制作用, 且毒副 作用小. 作用机理研究认为 ${ }^{[4,5,6]}$, 其通过与 DNA 作用、 止调 p53、破坏线粒体功能、阻碍葡萄糖吸收等方式, 诱 导肿瘤细胞凋亡. 但大黄酸的非水溶性及肿瘤细胞增殖
的中等抑制活性，使得其进一步的使用及研究均受到限 制. 因而研究其生物活性及机理, 对其进行结构改造, 以开发新型高效低毒抗肿瘤药物, 成为目前研究热点之 一, 并取得一定进展. 例如大黄酸与赖氨酸形成的一赖 氨大黄酸盐能够有效地改善大黄酸的水溶性, 从而提高 其抗肿瘤活性 ${ }^{[7]}$; Wang 等 ${ }^{[8]}$ 以大黄酸为原料合成了小分 子取代的酰胺大黄酸, 其抗肿瘤活性较大黄酸有了明显 提高; Wang 等 ${ }^{[9]}$ 首次用 $\alpha$-氨基磷酸酯修饰大黄酸, 得到 了一系列新型的葸醌衍生物. 大黄酸赖氨酸盐还可以与 5 -氟尿嘧啶发生协同的抗肿瘤活性, 是一种治疗人宫颈 癌的潜在的化学增敏药物 ${ }^{[10,11]}$. 另外, 研究发现, 氨基 葱醌衍生物, 例如酸性蓝 25 和酸性蓝 129 等(图 1), 除 用作染料外，可用于多种药物开发的先导化合物 ${ }^{[12,13]}$. 许多以 1-氨基-2-磺酸基-4-溴葱醌为原料, 合成抗肿瘤

\footnotetext{
*E-mail: lixl@hbu.cn

Received February 16, 2016; revised April 26, 2016; published online May 3, 2016.

Project supported by the National Natural Science Foundation of China (Nos. 21172051, 21372059).

国家自然科学基金(Nos. 21172051, 21372059)资助项目.
} 
药物的研究见诸报道 ${ }^{[14,15]}$.

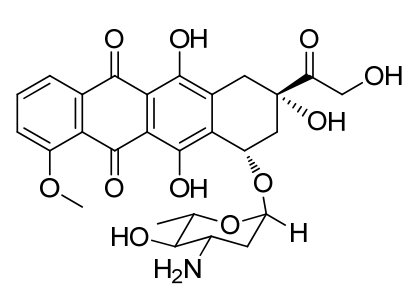<smiles>COc1cccc2c1C(=O)c1c(O)c3c(c(O)c1C2=O)C[C@@](C)(C(=O)CO)C[C@@H]3OC1CC(O)C(O)C1C</smiles>

Doxorubicin<smiles>Nc1c(S(N)(=O)=O)cc(Nc2ccccc2)c2c1C(=O)c1ccccc1C2=O</smiles>

Sabarubicin $\mathrm{O}$

Acid Blue 25<smiles>Cc1cc(C)c(Nc2cc(S(=O)(=O)O)c(N)c3c2C(=O)c2ccccc2C3=O)c(C)c1</smiles><smiles>O=C(O)c1cc(O)c2c(c1)C(=O)c1cccc(O)c1C2=O</smiles>

Acid Blue 129

图 1 部分葸醌衍生物结构

Figure 1 Structures of some anthraquinone derivatives

众所周知, 糖在生命系统中起着多种至关重要的作 用 ${ }^{[16]}$. 多种蒽醌类抗肿瘤药物中均含有糖的结构, 如前 面所涉及的阿霉素、表阿霉素、米托葸醌等. 氮杂糖(亚 胺糖)为糖分子成环氧原子被氮原子代替的糖模拟物, 是一类具有显著生物活性 ${ }^{[17]}$ 化合物, 特别是氮杂糖的 氮原子, 在生理环境下可以与 DNA 的磷酸酯链以静电 等形式相互作用, 以增强化合物分子与 DNA 的结合能 力, 从而提高化合物的抗肿瘤活性. 本文为开发高效低 毒蒽醌类抗肿瘤化合物, 在大黄酸及 1-氨基-4-溴蒽酮2-磺酸分子上引入氮杂糖, 改善化合物的水溶性的同 时, 增加化合物与 DNA 的相互作用, 以提高化合物的 抗肿瘤活性, 设计合成系列不同链长连接的氮杂糖修饰 的葸醌衍生物(图 2). 对所合成目标化合物 3 5, 7 9 进 行了初步体外抑制宫颈癌 Hela、肺癌 A549、乳腺癌 MCF-7 和胃癌 MGC-803 肿瘤细胞增殖的活性测试.<smiles>[R]C(=O)c1cc(O)c2c(c1)C(=O)c1c(O)cccc1C2=O</smiles>

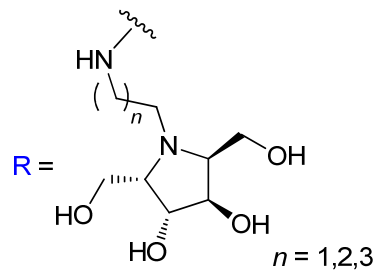

图 2 设计合成化合物结构

Figure 2 Structures of design and synthesis

\section{1 结果与讨论}

\section{1 化合物 3、4 和 5 的合成}

以甘露醇为原料 ${ }^{[18]}$, 经 1,3 和 4,6 羟基苯甲醛保护、 2、5 位羟基三氟甲磺酰基化, 然后分别与 $N$-叔丁氧羰 基-乙二胺、 $N$-叔丁氧羰基-丙二胺和 $N$-叔丁氧羰基-丁 二胺反应，生成相应的苯甲醛保护的 $N$-烷氨基氮杂糖， 经酸性条件下脱保护得化合物 $\mathbf{2 a} \sim \mathbf{2 c}$. 对大黄酸与化 合物 $\mathbf{2 a}$ 的缩合反应条件进行了探讨，部分数据列于表 1 中. 选用肽链合成常用的 2-(7-偶氮苯并三氮唑)$N, N, N^{\prime}, N^{\prime}$-四甲基嫝六氟磷酸酯(HATU)为缩合剂, 在碱 $N, N$-二异丙基乙胺(DIPEA)作用下，经反应物比例、温度 等条件探讨，选用表 1 中 Entry 5 对化合物 $\mathbf{3}$ 进行了合成， 即在 DMF 溶剂中, $\mathbf{1}: \mathbf{2 a}:$ HATU $:$ DIPEA $=1: 1.2$ : 2:1(物质的量比), 室温反应 $2 \mathrm{~h}$ 后, 反应液经常规处 理, 柱色谱分离, 得化合物 3 , 产率 $83 \%$. 在相同条件 下，大黄酸分别与化合物 $\mathbf{2 b}$ 和 $2 \mathrm{c}$ 反应，合成相应氮杂 糖修饰的大黄酸酰胺衍生物 4 (产率 79\%)和 5 (产率 85\%)(Eq. 1).

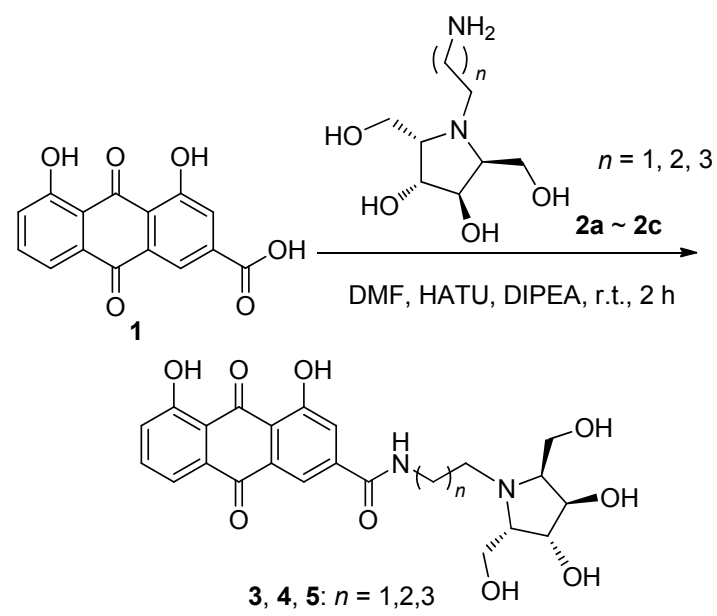

表 1 化合物 3 合成条件探讨

Table 1 Condition of the synthesis of compound 3

\begin{tabular}{ccccc}
\hline Entry & $\begin{array}{c}\mathbf{1}: \mathbf{2 a}: \text { HATU }: \text { DIPEA } \\
\text { (molar ratio) }\end{array}$ & $T /{ }^{\circ} \mathrm{C}$ & $t^{a} / \mathrm{h}$ & Yield/\% \\
\hline 1 & $1: 1: 1: 1$ & 0 & 5 & 20 \\
2 & $1: 1: 1: 1$ & r.t. & 5 & 75 \\
3 & $1: 1: 1: 1$ & 40 & 2 & 70 \\
4 & $1: 1.2: 1: 1$ & r.t. & 3 & 80 \\
5 & $1: 1.2: 2: 1$ & r.t. & 2 & 83 \\
6 & $1: 1.5: 2: 1$ & r.t. & 2 & 83 \\
\hline${ }^{a}$ TLC 监测原料 1 消失.
\end{tabular}

\section{2 化合物 $7 、 8$ 和 9 的合成}

采用微波促进 $\mathrm{Cu}^{0}$ 催化乌尔曼偶联(Ullmann coupling)反应 ${ }^{[19]}$, 对化合物 7 进行了合成(Eq. 2), 探讨了温 度、反应时间、反应物比例等对产率的影响，并与传统 
加热反应进行了对比. 部分数据列于表 2. 如表所示, 温度低时, 反应时间较长; 温度高, 则由于副产物增多, 而使产率下降. 氨基氮杂糖的比例低时, 产率亦有所下 降. 与传统加热反应相比, 微波促进不仅使反应时间大 大缩短 $(10 \mathrm{~h}$ 缩至 $15 \mathrm{~min}$ ), 同时使产率亦有所提高 $(70 \%$ 升至 76\%). 选用表 2 中 Entry 5 , 对化合物 7 进行了合成. 即在微波管中依次加入化合物 $6 、 \mathrm{pH}=6 \sim 7$ 的磷酸缓冲 溶液、 2 equiv.氮杂糖 $\mathbf{2 a}$ 、催化量的铜粉, 微波辅助下 $80{ }^{\circ} \mathrm{C}$ 反应 $15 \mathrm{~min}$, 经后处理, 得化合物 7 , 产率 $75 \%$. 在相同条件下, 化合物 $\mathbf{6}$ 分别与化合物 $\mathbf{2 b}$ 和 $\mathbf{2 c}$ 反应, 合 成相应氮杂糖修饰的葱醌衍生物 8 (产率 $81 \%$ ) 和 9 (产率 89\%)(Eq. 2).<smiles>Nc1c(S(=O)(=O)[O-])cc(Br)c2c1C(=O)c1ccccc1C2=O</smiles><smiles>NCCN1C(CO)C(O)C(O)C1CO</smiles><smiles>Nc1c(O[Na])cc(NC=CN2C(CO)[C@H](O)C(O)[C@H]2CO)c2c1C(=O)c1ccccc1C2=O</smiles>

7, 8, $9 n=1,2,3$

表 2 化合物 7 合成条件探讨

Table 2 Condition of the synthesis of compound 7

\begin{tabular}{cclcc}
\hline Entry & $\mathbf{6}: \mathbf{2 a}$ (molar ratio) & Conditions & $t /$ min & Yield $/ \%$ \\
\hline 1 & $1: 1$ & M.W., $70{ }^{\circ} \mathrm{C}$ & 25 & 61 \\
2 & $1: 1$ & M.W., $80{ }^{\circ} \mathrm{C}$ & 20 & 65 \\
3 & $1: 1$ & M.W., $90{ }^{\circ} \mathrm{C}$ & 15 & 60 \\
4 & $1: 1.5$ & M.W., $80{ }^{\circ} \mathrm{C}$ & 15 & 70 \\
5 & $1: 2$ & M.W., $80{ }^{\circ} \mathrm{C}$ & 15 & 75 \\
6 & $1: 2.5$ & M.W., $80{ }^{\circ} \mathrm{C}$ & 15 & 76 \\
7 & $1: 2$ & $120{ }^{\circ} \mathrm{C}$ & 600 & 70 \\
\hline
\end{tabular}

\section{3 化合物的细胞毒性}

利用 MTT 法, 以顺铂和盐酸阿霉素为阳性对照, 对所合成目标化合物 3 5 和 7 9 分别进行了体外抑制 宫颈癌 Hela、肺癌 A549、乳腺癌 MCF-7 和胃癌 MGC-803 肿瘤细胞的活性测试. 从活性数据(表 3)上看, 化合物没 有明显的肿瘤细胞抑制活性. 对所测三个细胞株, 所有 化合物对 Hela 细胞显示出一定的选择性, 尤其是四个 碳连接的氮杂糖修饰的大黄酸 $\mathbf{5}$, 对 Hela 的抑制活性明
显优于对肺癌 A549、乳腺癌 MCF-7 和 MGC-803 的抑 制活性, 具有进一步的结构改造及细胞毒性研究的潜 力. 化合物氮杂糖与葱醌环之间连接臂的长短, 对肿瘤 细胞的抑制活性有明显影响，对该类抗肿瘤化合物的设 计合成提供一定的依据.

表 3 化合物 $3 \sim 9$ 的体外肿瘤细胞毒性

Table 3 Cytotoxic activities of compounds $3 \sim 9$

\begin{tabular}{ccccc}
\hline \multirow{2}{*}{ Compd. } & \multicolumn{4}{c}{ Cytotoxicity $\left[\mathrm{IC}_{50}\left(\mu \mathrm{mol} \cdot \mathrm{L}^{-1}\right)\right]$} \\
\cline { 2 - 5 } & A549 & MCF-7 & Hela & MGC-803 \\
\hline $\mathbf{3}$ & 106.88 & 172.03 & 72.56 & 74.02 \\
$\mathbf{4}$ & 120.66 & 145.88 & 47.52 & 53.00 \\
$\mathbf{5}$ & 100.08 & 233.53 & 24.83 & 110.51 \\
$\mathbf{7}$ & 90.81 & 201.04 & 82.52 & 91.83 \\
$\mathbf{8}$ & 74.35 & 75.18 & 58.07 & 135.95 \\
$\mathbf{9}$ & 69.85 & 81.30 & 68.88 & 107.24 \\
顺铂 & 8.93 & 6.59 & 6.09 & 12.56 \\
盐酸阿霉素 & 1.52 & 2.78 & 0.38 & 1.43 \\
\hline
\end{tabular}

\section{2 结论}

为开发高效、低毒葱醌类抗肿瘤化合物, 以大黄酸 和 1-氨基-4-溴葱酮-2-磺酸为原料, 分别与 $N$-烷氨基氮 杂糖通过缩合反应、微波促进 $\mathrm{Cu}^{0}$ 催化乌尔曼偶联 (Ullmann coupling)反应，设计并合成系列新型氮杂糖修 饰的葱醌衍生物. 利用 MTT 法, 以顺铂和盐酸阿霉素 为阳性对照, 对所合成的化合物进行了初步体外肿瘤细 胞(Hela、A549、MCF-7 和 MGC-803)的细胞毒性测试, 化合物没有显示出明显的肿瘤细胞抑制活性. 初步分析 显示, 对所测肿瘤细胞株, 所有化合物对 Hela 细胞显示 出一定的选择性, 其中四个碳连接的氮杂糖修饰的大黄 酸 5, 对 Hela 的抑制活性明显优于对肺癌 A549、乳腺 癌 MCF-7 和 MGC-803 的抑制活性. 化合物氮杂糖与萝 醌环之间连接臂的长短, 对其对肿瘤细胞的抑制活性有 明显影响，对该类化合物的进一步设计合成提供一定的 依据。

\section{3 实验部分}

\section{1 仪器与试剂}

Bruker AC-P600 型核磁共振仪 (600 MHz)，四甲基 硅烷(TMS)为内标; FTICR-MS(Ionspec 7.0T)型质谱仪, ESI 离子源; Discover S-Class 微波化学合成系统(美国 CEM 公司); SGW-1 X-4 显微熔点仪, 上海精密科学仪 器有限公司.

柱层析用硅胶(200 300 目)及 GF254 薄层层析硅 胶为青岛海洋化工厂产品; 反相柱填料 $\left(\mathrm{C}_{18}\right)$ 由 $\mathrm{YMC}$ 公 司生产. 所用试剂和溶剂均为国产或进口(Aldrich 和 $\mathrm{TCI}$ 等公司)分析纯或化学纯试剂, 无水试剂均按常规 方法处理. 


\section{2 实验方法}

\subsection{1 化合物 $3 、 4$ 和 5 的合成}

$50 \mathrm{~mL}$ 圆底烧瓶中加入 $120 \mathrm{mg}(0.42 \mathrm{mmol})$ 大黄酸 1, $104 \mathrm{mg}(0.50 \mathrm{mmol}) 2 \mathrm{a}, 380 \mathrm{mg}(1.00 \mathrm{mmol}) 2$-(7-偶氮 苯并三氮唑)- $N, N, N^{\prime}, N^{\prime}$ - 四甲基艮六氟磷酸酯(HATU), 加入 $4 \mathrm{~mL}$ 新蒸 $\mathrm{DMF}$, 室温摚拌 $15 \mathrm{~min}$ 后, 加入 $110 \mu \mathrm{L}$ $N, N$-二异丙基乙胺(DIPEA), 继续室温搅拌 $2 \mathrm{~h}, \mathrm{TLC}$ 监 测反应完全. 用 $1 \mathrm{~mol} / \mathrm{L}$ 盐酸溶液中和至中性. 减压蒸 馏除去溶剂, 200 300 目硅胶柱层析, 淋洗剂( $V$ 二攻甲唍：

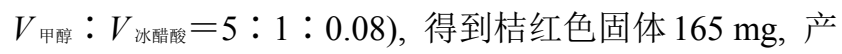
率为 $83 \%$. 按照该方法得化合物 4 (产率为 79\%)、5(产率 为 $85 \%$ ).

4,5-二羟基-2-( $N-2-((2 S, 3 R, 4 R, 5 S)-3,4$-二羟基- 2,5 -二 羟甲基吡咯啉基)乙基)葱醌甲酰胺(3): 桔红色固体, 产 率 $83 \%$. m.p. $250{ }^{\circ} \mathrm{C}$ (分解); ${ }^{1} \mathrm{H}$ NMR (600 MHz, $\left.\mathrm{CD}_{3} \mathrm{OD}\right)$ $\delta: 3.08 \sim 3.12(\mathrm{~m}, 1 \mathrm{H}, \mathrm{CH}), 3.42 \sim 3.44(\mathrm{~m}, 1 \mathrm{H}, \mathrm{CH})$, $3.69 \sim 3.71\left(\mathrm{~m}, 2 \mathrm{H}, \mathrm{CH}_{2}\right), 3.82 \sim 3.84(\mathrm{~m}, 1 \mathrm{H}, \mathrm{CH}), 3.86 \sim$ $3.88\left(\mathrm{~m}, 3 \mathrm{H}, \mathrm{CH}_{2}, \mathrm{CH}\right), 4.00 \sim 4.02\left(\mathrm{~m}, 4 \mathrm{H}, \mathrm{CH}_{2}\right), 4.27$ (d, $\left.J=2.4 \mathrm{~Hz}, 2 \mathrm{H}, \mathrm{CH}_{2}\right), 7.36(\mathrm{~d}, J=2.4 \mathrm{~Hz}, 1 \mathrm{H}, \mathrm{CH}), 7.77 \sim$ 7.81 (m, 3H, CH), $8.20(\mathrm{~m}, 1 \mathrm{H}, \mathrm{CH}) ;{ }^{13} \mathrm{C}$ NMR $(150 \mathrm{MHz}$, DMSO- $\left.d_{6}\right) \delta: 23.97,29.30,33.90,39.17,46.11,48.52$, $53.90,57.93,117.55,119.26,120.08,121.40,124.38$, $126.30,133.10,138.59,142.48,161.86,162.93,173.15$, 177.98, 178.20. HRMS calcd for $\mathrm{C}_{23} \mathrm{H}_{24} \mathrm{~N}_{2} \mathrm{O}_{9} 472.1482$, found 472.1485 .

4,5-二羟基-2-( $N-3$ - $((2 S, 3 R, 4 R, 5 S)-3,4$-二羟基-2,5-二 羟甲基吡咯啉基)丙基)葱醌甲酰胺(4): 桔红色固体, 产 率 79\%. m.p. $250{ }^{\circ} \mathrm{C}$ (分解); ${ }^{1} \mathrm{H}$ NMR (600 MHz, $\left.\mathrm{CD}_{3} \mathrm{OD}\right)$ $\delta: 2.10 \sim 2.14\left(\mathrm{~m}, 2 \mathrm{H}, \mathrm{CH}_{2}\right), 2.21 \sim 2.23(\mathrm{~m}, 1 \mathrm{H}, \mathrm{CH}), 3.37$ (s, $1 \mathrm{H}, \mathrm{CH}), 3.42 \sim 3.44(\mathrm{~m}, 1 \mathrm{H}, \mathrm{CH}), 3.57 \sim 3.60(\mathrm{~m}, 2 \mathrm{H}$, $\left.\mathrm{CH}_{2}\right), 3.91(\mathrm{~s}, 1 \mathrm{H}, \mathrm{CH}), 3.99 \sim 4.01\left(\mathrm{~m}, 2 \mathrm{H}, \mathrm{CH}_{2}\right), 4.26(\mathrm{~s}$, $\left.2 \mathrm{H}, \mathrm{CH}_{2}\right), 7.36(\mathrm{~s}, 1 \mathrm{H}, \mathrm{CH}), 7.72 \sim 7.83(\mathrm{~m}, 2 \mathrm{H}, \mathrm{CH}), 8.20$ $(\mathrm{s}, 1 \mathrm{H}, \mathrm{CH}), 8.54(\mathrm{~s}, 1 \mathrm{H}, \mathrm{CH}) ;{ }^{13} \mathrm{C}$ NMR $(150 \mathrm{MHz}$, DMSO- $\left.d_{6}\right) \delta: 21.31,26.10,29.27,37.51,38.60,49.37$, 57.12, 68.13, 75.87, 79.04, 79.17, 119.41, 119.71, 127.11, $129.72,130.06,137.15,137.55,147.28,153.34,158.44$, 162.89 , 171.61, 178.08, 179.13. HRMS calcd for $\mathrm{C}_{24} \mathrm{H}_{26} \mathrm{~N}_{2} \mathrm{O}_{9} 486.1638$, found 486.1643 .

4,5-二羟基-2-( $N-4-((2 S, 3 R, 4 R, 5 S)-3,4$-二羟基-2,5-二 羟甲基吡咯啉基)丁基)葱醌甲酰胺(5): 桔红色固体, 产 率 $85 \%$. m.p. $251{ }^{\circ} \mathrm{C}$ (分解), ${ }^{1} \mathrm{H}$ NMR (600 MHz, $\left.\mathrm{CD}_{3} \mathrm{OD}\right)$ $\delta: 1.42 \sim 1.48\left(\mathrm{~m}, 2 \mathrm{H}, \mathrm{CH}_{2}\right), 2.35 \sim 2.36\left(\mathrm{~m}, 2 \mathrm{H}, \mathrm{CH}_{2}\right)$, $3.40 \sim 3.44(\mathrm{~m}, 1 \mathrm{H}, \mathrm{CH}), 3.49\left(\mathrm{t}, J=4.8 \mathrm{~Hz}, 2 \mathrm{H}, \mathrm{CH}_{2}\right)$, $3.54 \sim 3.58(\mathrm{~m}, 1 \mathrm{H}, \mathrm{CH}), 4.01\left(\mathrm{~s}, 2 \mathrm{H}, \mathrm{CH}_{2}\right), 4.03(\mathrm{~s}, 1 \mathrm{H}$, $\mathrm{CH}), 4.05 \sim 4.09\left(\mathrm{~m}, 2 \mathrm{H}, \mathrm{CH}_{2}\right), 4.29 \sim 4.32\left(\mathrm{~m}, 2 \mathrm{H}, \mathrm{CH}_{2}\right)$, $7.36(\mathrm{~d}, J=6.6 \mathrm{~Hz}, 1 \mathrm{H}, \mathrm{CH}), 7.73 \sim 7.81(\mathrm{~m}, 3 \mathrm{H}, \mathrm{CH})$, $8.18(\mathrm{~s}, 1 \mathrm{H}, \mathrm{CH}) ;{ }^{13} \mathrm{C}$ NMR (150 MHz, DMSO- $\left.d_{6}\right) \delta$ : $19.29,22.96,26.28,29.25,39.12,46.09,52.33,56.54$, $69.48,71.61,75.31,117.50,119.55,127.26,131.10$, 133.60, 134.03, 134.38, 134.67, 136.72, 137.28, 166.27, 172.38, 181.45. HRMS calcd for $\mathrm{C}_{25} \mathrm{H}_{28} \mathrm{~N}_{2} \mathrm{O}_{9} 500.1795$, found 500.1791 .

\subsection{2 化合物 7、8 和 9 的合成}

于 $10 \mathrm{~mL}$ 的微波管中依次加入 $100 \mathrm{mg}$ 化合物 6、 $\mathrm{pH}=6 \sim 7$ 的磷酸缓冲溶液 $3 \mathrm{~mL} 、 100 \mathrm{mg}$ (2 equiv.)氮杂 糖 2a, 加入催化量的铜粉. 微波辅助下 $80{ }^{\circ} \mathrm{C}$ 反应 15 min. TLC 监测原料反应完全, 旋蒸蒸除溶剂, $\mathrm{C}_{18}$ 柱层析 分离得到蓝色固体 $798 \mathrm{mg}$, 产率 75\%. 按照该方法可 得到化合物 8(产率 81\%)、9(产率 89\%).

1-氨基-4- $N$ - $(2-((2 S, 3 R, 4 R, 5 S)-3,4$-二羟基- 2,5 -二羟 甲基吡咯啉基)乙基)氨基-2-葱醌磺酸钠(7): 蓝色固体, 产率 75\%. m.p. 278 $279{ }^{\circ} \mathrm{C} ;{ }^{1} \mathrm{H}$ NMR $(600 \mathrm{MHz}$, DMSO- $\left.d_{6}\right) \delta: 2.87 \sim 2.93(\mathrm{~m}, 1 \mathrm{H}, \mathrm{CH}), 3.07(\mathrm{t}, J=6.6 \mathrm{~Hz}$, $\left.2 \mathrm{H}, \mathrm{CH}_{2}\right), 3.15$ (d, $\left.J=10.8 \mathrm{~Hz}, 1 \mathrm{H}, \mathrm{CH}\right), 3.23 \sim 3.24(\mathrm{~m}$, $\left.2 \mathrm{H}, \mathrm{CH}_{2}\right), 3.48 \sim 3.81\left(\mathrm{~m}, 2 \mathrm{H}, \mathrm{CH}_{2}\right), 3.51(\mathrm{~s}, 1 \mathrm{H}, \mathrm{CH})$, 3.67 (q, $\left.J=18 \mathrm{~Hz}, 2 \mathrm{H}, \mathrm{CH}_{2}\right) .7 .77$ (s, $\left.1 \mathrm{H}, \mathrm{CH}\right), 7.83 \sim 7.84$ (m, $\left.2 \mathrm{H}, \mathrm{CH}_{2}\right), 8.24 \sim 8.28\left(\mathrm{~m}, 2 \mathrm{H}, \mathrm{CH}_{2}\right) ;{ }^{13} \mathrm{C}$ NMR $(150$ $\mathrm{MHz}$, DMSO- $\left.d_{6}\right) \quad \delta: 109.93,110.47,120.96,126.21$, 133.20, 134.21, 143.52, 144.88, 181.98, 182.32. HRMS calcd for $\mathrm{C}_{22} \mathrm{H}_{24} \mathrm{~N}_{3} \mathrm{NaO}_{9} \mathrm{~S} 529.1131$, found 529.1127 .

1-氨基-4- $N-(3-((2 S, 3 R, 4 R, 5 S)-3,4-$ 二羟基- $2,5-$ 二羟 甲基吡咯啉基)丙基)氨基-2-葱醌磺酸钠(8): 蓝色固体, 产率 $81 \%$. m.p. $285 \sim 286{ }^{\circ} \mathrm{C} ;{ }^{1} \mathrm{H}$ NMR $(600 \mathrm{MHz}$, DMSO- $\left.d_{6}\right) \delta: 1.62 \sim 1.70(\mathrm{~m}, 1 \mathrm{H}, \mathrm{CH}), 1.96(\mathrm{q}, 2 \mathrm{H}, J=7.2$ $\left.\mathrm{Hz}, \mathrm{CH}_{2}\right), 2.50$ (s, 4H, $\left.\mathrm{CH}_{2}\right), 2.76(\mathrm{t}, J=7.2 \mathrm{~Hz}, 1 \mathrm{H}, \mathrm{CH}$ ), $3.06 \sim 3.10(\mathrm{~m}, 1 \mathrm{H}, \mathrm{CH}), 3.35(\mathrm{dd}, 1 \mathrm{H}, J=7.2,21.6 \mathrm{~Hz}$, $1 \mathrm{H}, \mathrm{CH}), 3.51\left(\mathrm{t}, J=6.6 \mathrm{~Hz}, 2 \mathrm{H}, \mathrm{CH}_{2}\right), 7.77(\mathrm{~s}, 1 \mathrm{H}, \mathrm{CH})$, $7.80 \sim 7.82(\mathrm{~m}, 2 \mathrm{H}, \mathrm{CH}), 8.22 \sim 8.23(\mathrm{~m}, 1 \mathrm{H}, \mathrm{CH}), 8.24 \sim$ $8.26(\mathrm{~m}, 1 \mathrm{H}, \mathrm{CH}) ;{ }^{13} \mathrm{C}$ NMR $\left(150 \mathrm{MHz}\right.$, DMSO- $\left.d_{6}\right) \delta$ : $22.20,26.60,27.65,31.70,37.11,42.57,64.70,73.18$, $109.84,121.51,126.19,126.41,133.24,134.32,143.09$, $145.14,160.53,173.66,181.84,182.39$. HRMS calcd for $\mathrm{C}_{23} \mathrm{H}_{26} \mathrm{~N}_{3} \mathrm{NaO}_{9} \mathrm{~S} 543.1287$, found 543.1284.

1-氨基-4- $N$-(4-((2S,3R,4R,5S)-3,4-二羟基-2,5-二羟 甲基吡咯啉基)丁基)氨基-2-葱醌磺酸钠(9): 蓝色固体, 产率 89\%. m.p. $>300{ }^{\circ} \mathrm{C},{ }^{1} \mathrm{H}$ NMR $(600 \mathrm{MHz}$, DMSO$\left.d_{6}\right) \delta: 1.48 \sim 1.53\left(\mathrm{~m}, 2 \mathrm{H}, \mathrm{CH}_{2}\right), 1.98 \sim 2.02\left(\mathrm{~m}, 2 \mathrm{H}, \mathrm{CH}_{2}\right)$, $2.61 \sim 2.62\left(\mathrm{~m}, 2 \mathrm{H}, \mathrm{CH}_{2}\right), 3.27 \sim 3.30(\mathrm{~m}, 1 \mathrm{H}, \mathrm{CH}), 3.42 \sim$ $3.43\left(\mathrm{~m}, 2 \mathrm{H}, \mathrm{CH}_{2}\right), 3.49 \sim 3.49(\mathrm{~m}, 1 \mathrm{H}, \mathrm{CH}), 3.86(\mathrm{~s}, 1 \mathrm{H}$, $\mathrm{CH}), 3.98(\mathrm{t}, J=3.0 \mathrm{~Hz}, 1 \mathrm{H}), 4.18(\mathrm{~d}, J=5.0 \mathrm{~Hz}, 2 \mathrm{H}$, 
$\left.\mathrm{CH}_{2}\right), 7.50(\mathrm{~s}, 1 \mathrm{H}, \mathrm{CH}), 7.59 \sim 7.61(\mathrm{~m}, 2 \mathrm{H}, \mathrm{CH}), 8.01 \sim$ $8.02(\mathrm{~m}, 2 \mathrm{H}, \mathrm{CH}) ;{ }^{13} \mathrm{C}$ NMR $\left(150 \mathrm{MHz}, \mathrm{DMSO}-d_{6}\right) \delta$ : $23.07,24.87,25.05,26.57,34.45,38.27,38.86,42.07$, $55.45,73.38,81.46,109.36,121.34,126.16,126.37$, $133.08,134.35,143.43,145.62,181.24,182.08$. HRMS calcd for $\mathrm{C}_{24} \mathrm{H}_{28} \mathrm{~N}_{3} \mathrm{NaO}_{9} \mathrm{~S} 557.1444$, found 557.1449.

\subsection{3 细胞毒性实验}

抗肿瘤细胞毒性采用改进的 Mosmann's MTT 染色 法测定 ${ }^{[20]}$. 具体操作如下: 于 96 孔板接种细胞悬液, 90 $\mu \mathrm{L} /$ 孔, 细胞密度 $2 \times 104$ 个 $/ \mathrm{mL}, 37^{\circ} \mathrm{C}, 5 \% \mathrm{CO}_{2}$ 浓度的 培养箱中培养过夜, 加 $10 \mu \mathrm{L} /$ 孔相应浓度药物(100 $\mu$ $\mathrm{mol} / \mathrm{L}$ ), 继续培养 $44 \mathrm{~h}$, 加 MTT $10 \mu \mathrm{L} /$ 孔培养 $4 \mathrm{~h}$, 从培 养箱中取出, 移去上清液, 加 DMSO $100 \mu \mathrm{L} /$ 孔溶解 MTT, 测量 $570 \mathrm{~nm}$ 波长下肿瘤细胞 OD 值, 代入公式: 抑制率 $(\%)=\left(\mathrm{OD}_{\text {control }}-\mathrm{OD}_{\text {treated }}\right) / \mathrm{OD}_{\text {control }} \times 100 \%$ 计算.

\section{辅助材料(Supporting Information) 所合成化合物的} ${ }^{1} \mathrm{H}$ NMR、 ${ }^{13} \mathrm{C}$ NMR 谱图. 这些材料可以免费从本刊网 站(http://sioc-journal.cn/)上下载.

\section{References}

[1] Monneret, C. Eur. J. Med. Chem. 2001, 36, 483.

[2] Buzdar, A. U.; Marcus, C.; Smith, T. L.; Blumenschein, G. R. Cancer 1985, 55, 2761 .

[3] Bolger, T. A.; Yao, T. P. J. Neurosci. 2005, 25, 9544.

[4] Duraipandiyan, V.; Baskar, A. A.; Ignacimuthu, S.; Muthukumar, C.
Al-Harbi, N. A. Asian Pac. J. Trop. Dis. 2012, 517.

[5] Huang, Q.; Lu, G.; Shen, H. M.; Cbung, M. C. M.; Ong, C. N. Med. Res. Rev. 2007, 27, 609.

[6] Panigrahi, G. K.; Yadav, A.; Srivastava, A.; Tripathi, A.; Raisuddin, S.; Das, M. Chem. Res. Toxicol. 2015, 28, 1133.

[7] Lin, Y. J.; Zhen, Y. S. Anti-cancer Drugs 2009, 20, 65.

[8] Yang, X.; Sun, G.; Yang, C.; Wang, B. H. ChemMedChem 2011, 6, 2294.

[9] Yao, G. Y.; Ye, M. Y.; Huang, R. Z.; Li, Y. J.; Pan, Y. M.; Xu, Q.; Liao, Z. X.; Wang, H. S. Bioorg. Med. Chem. Lett. 2014, 24, 501.

[10] Zhang, Z. H.; Yamashita, H.; Toyama, T.; Sugiura, H.; Omoto, Y.; Ando, Y.; Mita, K.; Hamaguchi, M.; Hayashi, S. I.; Iwase, H. Clin. Cancer Res. 2004, 10, 6962;

[11] Aldana-Masangkay, G. I.; Sakamoto, K. M. J. Biomed. Biotechnol. 2010, 2011.

[12] Glänzel, M.; Bültmann, R.; Starke, K.; Frahm, A. W. Eur. J. Med. Chem. 2003, 38, 303.

[13] Glänzel, M.; Bültmann, R.; Starke, K.; Frahm, A. W. Eur. J. Med. Chem. 2005, 40, 1262.

[14] Baqi, Y.; Atzler, K.; Köse, M.; Glänzel, M.; Müller, C. E. J. Med. Chem. 2009, 52, 3784.

[15] Baqi, Y.; Lee, S. Y.; Iqbal, J.; Ripphausen, P.; Lehr, A.; Scheiff, A. B.; Zimmermann, H.; Bajorath, J.; Müller, C. E. J. Med. Chem. 2010, 53, 2076.

[16] Sears, P.; Wong, C. H. Angew. Chem., Int. Ed. 1999, 38, 2300.

[17] Compain, P.; Martin, O. R. Iminosugars: from Synthesis to Therapeutic Applications, John Wiley and Sons, Ltd., Chichester, 2007, p. 87.

[18] Zhang, P. Z.; Yang, H. L.; Li, C. C.; Xia, Z. C.; Wang, X. M.; Wei, H.; Rong, R. X.; Cao, Z. R.; Wang, K. R.; Chen, H.; Li, X. L. Chin. Chem. Lett. 2014, 25, 1057.

[19] Baqi, Y.; Muller, C. E. Org. Lett. 2007, 9, 1271.

[20] Mosmann, T. J. Immunol. Methods 1983, 65, 55.

(Cheng, F.) 